Evolución de la oferta exportable en Santander, Colombia 2000-2012:

Un análisis a través del índice Herfindahl Hirschmann

Evolution of exportable supply in Santander, Colombia 2000-2012:

An analysis through the Herfindahl Hirschmann Index. 


\title{
Evolución de la oferta exportable en Santander, Colombia 2000-2012: Un análisis a través del índice Herfindahl Hirschmann ${ }^{1}$
}

\section{Evolution of exportable supply in Santander, Colombia 2000-2012: An analysis through the Herfindahl Hirschmann Index.}

\author{
Estefani Prada Villamizar ${ }^{2}$, Gustavo García Cediel$^{3}$, Ricardo Andrade Díaz ${ }^{4}$ \\ Universidad de Valencia, España.
}

Artículo recibido en diciembre de 2015; artículo aceptado en mayo de 2016

Citación del artículo: Prada, E., García, G. \& Andrade, R. (2016).Evolución de la oferta exportable en Santander, Colombia 2000-2012: Un análisis a través del índice Herfindahl Hirschmann. I+D Revista de Investigaciones, 7(1), 78-90.

\begin{abstract}
Resumen
Este documento tuvo como objetivo analizar la evolución de la oferta exportadora de Santander entre el año 2000 y 2012, revisando los patrones de concentración o diversificación. Para ello utilizó como metodología el cálculo del índice de Herfindahl-Hirschmann. Los hallazgos encontrados establecen que la oferta exportable se ha concentrado por lo que las políticas diseñadas para promover la diversificación de producto no han tenido los efectos esperados y es necesario el diseño de nuevos lineamientos para tal fin.
\end{abstract}

Palabras Clave: Internacionalización, comercio exterior, concentración exportadora, diversificación exportadora, crecimiento económico, índice de HerfindahlHirschmann.

\section{Abstract}

This paper aimed to analyze the evolution of the export supply of Santander between 2000 and 2012 reviewing the concentration or diversification patterns. For this, the methodology used was the index Herfindahl - Hirschmann. The findings establish that the export supply is concentrated so that policies designed to promote product diversification have not had the desired effect and is necessary to design new guidelines for this purpose.

Keywords: Internationalization, foreign trade, export concentration, Herfindahl- Hirschmann index.

\section{Introducción}

Posturas teóricas como la Teoría de la Dependencia (Prebisch, 1950) consideran que los países deben pasar de una estructura productiva basada en la explotación y exportación de los recursos naturales hacia la manufactura de bienes industriales con alto valor agregado con el fin de no verse afectados por el deterioro usual en los términos de intercambio $\mathrm{y}$, de esa manera, generar mayores tasas de crecimiento y, por ende, mayores tasas de empleo y bienestar para la población.

De acuerdo a esto, Latinoamérica en los inicios del siglo

${ }^{1}$ Artículo de investigación con enfoque cuantitativo, trabajo derivado de la tesis "Propuesta de lineamientos de política pública para el fomento de la diversificación de las exportaciones santandereanas en productos y destinos" presentada por Estefani Prada Villamizar para obtener el título de magíster en administración de la Universidad Santo Tomas y terminado de ajustar por los grupos de investigación PORTER y SECLAM, de la ciudad de Bucaramanga (Colombia). Calle 9 no 2355, PBX: 6352525. Fecha de inicio: Junio 2013, fecha de terminación: Febrero de 2015.

${ }^{2}$ Candidata a doctora en dirección de empresas de la Universidad de Valencia. Magister en administración de empresas, Universidad Santo Tomas. Economista de la Universidad Industrial de Santander. Correo electrónico: espravi@alumni.uv.es

${ }^{3}$ Candidato a doctor en dirección de empresas de la Universidad de Valencia. Magíster en Economía de la Pontificia Universidad Javeriana. Especialista en Finanzas con énfasis en Banca de Inversión de la Universidad Externado de Colombia. Economista de la Universidad Industrial de Santander. Docente tiempo completo Universidad Cooperativa de Colombia. Seccional Bucaramanga calle 30ª No 33-51. Email: gustavo.garciac@campusucc.edu.co

${ }^{4}$ Magíster en Gerencia de Empresas Mención Mercadeo, Universidad Nacional Experimental del Táchira. Administrador de Negocios de la Universidad de San Buenaventura, Bogotá. Docente de tiempo completo de la Corporación de Investigación y Desarrollo, UDI. Calle 9\#23-55. Correo electrónico: randrade1@udi.edu.co 
XX, giró hacia un modelo de desarrollo basado en la Industrialización por Sustitución de Importaciones (ISI) y al final de ese periodo, ante el agotamiento de dicho modelo, se dio inicio a un proceso de apertura pasando de modelos cerrados a modelos abiertos al comercio internacional; estos modelos buscan diversificar la oferta exportable y sus destinos, por ello ha sugerido a distintas naciones de Suramérica a buscar socios comerciales que demanden sus productos y les permita disminuir los efectos sugeridos por la Teoría de la Dependencia así como llevar a cabo procesos de reconversión tecnológica que permitan producir bienes que incorporen mayores niveles de tecnología.

Colombia no ha sido la excepción a esta postura, y el país inició un proceso para abrirse al mercado internacional identificándose dos etapas: una inicial, a principios de los años 90 cuando realizó principalmente reformas en la política comercial disminuyendo aranceles, y una segunda etapa, basada en la firma de múltiples acuerdos de libre comercio pasando de contar con tres acuerdos en el año 2000 a 22 en el año 2014. A nivel regional, los territorios también han seguido este camino y Santander, uno de sus principales departamentos por participación en el PIB, ha establecido a la internacionalización como uno de los pilares de su Plan de Competitividad y el tema ha sido incluido en sus últimos Planes de Desarrollo. Sin embargo, no existen estudios técnicos sobre cómo dichos procesos de apertura han impactado la oferta exportadora de Santander

En este orden de ideas, este documento tiene como objetivo llenar ese vacío e indagar, mediante el cálculo de un índice estadístico de concentración como es el índice Herfindahl-Hirschmann, qué ha sucedido con la oferta exportadora de Santander durante el periodo 20002012.Desde el punto de vista gubernamental, alcanzar el objetivo propuesto, permitirá formular lineamientos adecuados de política pública según la situación que se encuentre.

El documento está organizado de la siguiente manera: en la primera sección se revisa la literatura sobre diversificación/concentración exportadora; posterior a ello, se presenta el diseño metodológico que se utilizó y finalmente, se discuten los resultados de la aplicación del índice para el Departamento.

\section{Revisión de la literatura}

El debate teórico respecto al grado de conveniencia que representa para una nación especializarse en la exportación de productos primarios data desde la década de 1950 con las investigaciones de Prebisch (1950) y Singer (1950) quienes advirtieron acerca del deterioro de los términos de intercambio que sufren aquellas naciones cuya canasta exportadora se compone básicamente de recursos naturales; al argumentar que dicha especialización productiva redunda con el tiempo, en que cada unidad de éstos va a comprar menores cantidades de bienes industriales. Lo anterior, profundiza la condición de subdesarrollo de estas economías, en tanto que la riqueza de los países desarrollados, medida en términos de su PIB per cápita, se incrementa constantemente (Prebisch, 1950).

Dicha situación, tiende a agravarse cuando los precios internacionales de los recursos naturales caen, especialmente en épocas de crisis, pues aquellas economías, generalmente se suman en profundas recesiones con grandes y lesivas consecuencias para su crecimiento futuro; condición que se agrava a causa de la alta dependencia por los recursos provenientes de la exportación de este tipo de bienes (Prebisch, 1950).

De lo anterior, se deriva la Teoría de la Dependencia, la cual afirma que, producto de la especialización de las exportaciones en recursos naturales, las economías, especialmente las subdesarrolladas, focalizan su aparato productivo en mejorar su obtención y comercialización, dejando de lado el desarrollo de otros sectores económicos como el industrial o el tecnológico, lo que acarrea consigo una fuerte dependencia tanto de los bienes que exportan y sus precios de mercado, como de los países con quienes comercian, que generalmente son naciones con altos niveles de desarrollo industrial y tecnológico; condiciones que no sólo les otorga poder de mercado, sino también económico y político, con lo que los naciones subdesarrolladas vienen a constituirse en dependientes de las decisiones que toman estos países en todos los sentidos, limitando por esta vía su marco de acción y por ende, sus niveles de bienestar y desarrollo en el mediano y largo plazo (Prebisch, 1950).

Estos planteamientos se condensan de una corriente teórica que se conoce como Pesimismo Exportador, la cual señala que para especializar las exportaciones de un país en bienes primarios, los recursos provenientes de su comercialización deben invertirse en la producción a gran escala y la adquisición de tecnologías para su extracción y tratamiento, que sólo producen los países desarrollados, los cuales, en primera instancia, son compradores de sus recursos naturales, pero que en un segundo momento del tiempo, se convierten en únicos proveedores que se quedan con gran parte de la riqueza de estas naciones, por lo que la actividad exportadora presenta un cuello de botella en términos financieros que impide utilizar los dineros de bonanzas y altos precios para el desarrollo de nuevas ventajas competitivas en otros sectores e incursionar en otros mercados, limitando con ello su capacidad de romper el ciclo del subdesarrollo.

En la misma línea, los trabajos de Krugman (1987) y Grossman \& Helpman (1991) muestran que un país que permite que su aparato productivo se concentre en exportaciones de recursos naturales, con el tiempo, queda condicionado a seguir produciendo los mismos bienes y a tener menores tasas de crecimiento, debido a que se obvia la innovación tecnológica que se da principalmente en el sector industrial.

Sachs \& Warner (1997) y Larraín et.al (1999) llaman a este fenómeno "La maldición de los recursos naturales", al 
evidenciar empíricamente que para los países subdesarrollados, la abundancia en estos recursos se convierte con el tiempo en un obstáculo para su crecimiento económico. De hecho, sus investigaciones aplicadas a 80 países en desarrollo, encontraron una relación inversa entre el índice de concentración de las exportaciones en recursos naturales y las tasas de crecimiento, medida en términos de su PIB, advirtiendo que cuando este tipo de naciones mantienen su oferta exportable basada en bienes primarios, enfrentan indefectiblemente una importante disminución de su crecimiento económico (Sachs \& Warner, 1997; Larraín et al, 1999).

En suma, las conclusiones más contundentes acerca de la "maldición de los recursos naturales" son, en primer lugar, que la especialización en estos recursos afecta negativamente el crecimiento económico en el largo plazo. Y también, que la concentración en este tipo de canasta exportadora no genera ningún vínculo fuerte con la innovación tecnológica, en términos de la inversión en investigación y desarrollo de las mismas, siendo esto nefasto para el desarrollo económico (Meller, 2002).

Los trabajos más recientes en este tema, analizan lo que se conoce como el "Síndrome Holandés" o la "Enfermedad Holandesa"; fenómeno que se da cuando un boom de exportaciones de recursos naturales o commodities genera para un país un gran flujo de divisas que aprecia su tasa de cambio, dejando en una situación de vulnerabilidad a los demás sectores exportadores. Así, la pérdida de competitividad de dichos sectores, irradia un efecto negativo a toda la economía, debilitándola y deprimiendo sus tasas de crecimiento (Sachs \& Warner 1997; Torvik, 2001).

En efecto, la teoría del "Síndrome Holandés" argumenta que las economías no deben fundamentar su crecimiento exportador en base a los recursos naturales pues, la existencia de grandes rentasen este sector, acarrea consigo un comportamiento rentista de los productores que frena una mayor inversión en otras actividades que resultan más rentables para las economías en términos de crecimiento. Asimismo, esas grandes rentas propician un ambiente que facilita acciones corruptas en el Gobierno para privilegiar el acceso de los recursos naturales a ciertas empresas, incluso extranjeras. De otra parte, la alta volatilidad de los precios internacionales de estos recursos trae consigo grandes desequilibrios macroeconómicos que afectan directamente los niveles de crecimiento económico de los países exportadores (World Bank, 2002; Maloney, 2002).

En el marco de este debate, un aspecto importante que señala la literatura es que el grado de afectación negativa o positiva que puede experimentar una economía que se especializa en la exportación de recursos naturales, está directamente asociado a la calidad de sus instituciones. En efecto, los trabajos más recientes señalan que es la calidad de éstas las que determinan el uso final de los recursos derivados de este tipo de actividad exportadora (Acemoglu et al, 2003; Hausman et al, 2007).

De acuerdo con Robinson et al (2006) quienes desarrollaron un modelo para medir la incidencia de las instituciones políticas en el grado en que se desarrolla la maldición de los recursos naturales en los países, las naciones con mayores índices de ineficiencia, burocracia y corrupción, presentan una mayor tendencia a administrar de forma incorrecta tanto la explotación como los recursos provenientes de la comercialización de los bienes primarios, por lo que un auge exportador en este sector redunda en una pérdida de competitividad de toda la economía en el mediano y largo plazo.

En la misma línea, Mehlum et al (2006), desarrollan un modelo económico en dos etapas en el que estiman el impacto de la especialización en recursos naturales sobre el crecimiento económico. Así, en la primera fase del estudio, los autores confirman que efectivamente la concentración de la canasta exportable en bienes primarios afecta negativamente el crecimiento económico, medido en términos del PIB. Sin embargo, en la segunda etapa, los autores encuentran que la calidad de las instituciones en interacción con la especialización en bienes primarios, tiene una relación directamente proporcional. Es decir, que ante una baja calidad institucional, la especialización en recursos naturales resulta en una verdadera maldición para las economías. Y por el contrario, una alta calidad de las instituciones hace de la abundancia de recursos naturales en los países, una verdadera bendición económica.

Si bien no existe aún una conclusión contundente, el debate continúa vigente principal mente a causa de la coyuntura económica de la primera década del siglo XXI en la que los precios de las materias primas han experimentado importantes tendencias alcistas en el mercado internacional, en tanto que los productos industrializados han sufrido una situación contraria. Pese a ello, la concentración de las exportaciones en bienes primarios aún preocupa y la diversificación de mercados con un componente de valor agregado hace parte de las agendas políticas de los Gobiernos y de los temas de investigación de la academia. De hecho, los actuales proyectos de apoyo a las exportaciones en países como Nueva Zelanda, Australia, Chile, Brasil y Colombia, incluyen una línea específica para la diversificación de la oferta exportable (Di Paula et al, 2008).

Así las cosas, el tema de la diversificación exportadora, tanto en productos como en destinos, es importante en la medida que estas características del crecimiento exportador son relevantes para el desarrollo de las economías, especialmente para aquellas pequeñas y abiertas al mercado mundial, en donde el crecimiento económico debe ir atado al comportamiento del sector exportador (Basal et al, 2011). De esa forma, al utilizar su riqueza para construir nuevas ventajas competitivas y promover la diversificación de las exportaciones, a través de esfuerzos gubernamentales por desarrollar e impulsar otros sectores de la economía, los 
países con alta dotación en recursos naturales, pueden valerse de su abundancia para desarrollar nuevas líneas que impulsen su crecimiento económico. (Bonaglia \& Fukasaku, 2003; Ferranti, 2001).

Ahora bien, desde el punto de vista técnico, para calcular el grado de concentración de la canasta exportadora de los países, el Índice de Herfindahl Hirschmann (IHH) ha sido utilizado en la literatura sobre economía industrial como indicador de la concentración/diversificación de los mercados. Esta medida funciona ponderando el peso de cada producto y país en el total de su comercio, teniendo la particularidad de que si el valor exportado es reducido, tiene una influencia pequeña en el indicador final, y viceversa. Así, se constituye en el principal referente técnico y científico para la medición de este fenómeno en las economías del mundo.

Con base en éste y otros indicadores, autores como Herzer \& Nowak-Lehmann (2006) establecen un vínculo directo y positivo entre la diversificación de la canasta exportadora y el crecimiento económico, a través de lo que denominan externalidades de aprendizaje. Así, de acuerdo con los resultados de su investigación, posicionar más sectores de la economía en el sector externo es mucho más importante para el crecimiento que intensificar las exportaciones de uno sólo.

En concordancia con esta tesis, Hausmann \& Rodrik (2003) sostienen que en los países subdesarrollados, el crecimiento está vinculado a la capacidad del sector empresarial para identificar la estructura de costos de una nueva actividad. En este sentido, estos países no diversifican su oferta exportadora, a causa de externalidades de información que impiden que los empresarios incursionen en nuevas actividades, en especial por el alto riesgo individual que deben asumir los pioneros. Sin embargo, con el apoyo suficiente del Gobierno, los empresarios pueden ser impulsados y coordinados para lanzarse a una nueva actividad productiva fácilmente imitable, de la cual puedan entender rápidamente su estructura de costos. De esa forma, el mismo mercado arrastra a los imitadores y con ello, los beneficios de la diversificación se irradian a toda la economía. Así, cuanto más crezca el número de empresarios incentivados a incursionar en una nueva actividad exportadora, más cerca está la economía de su frontera de productividad y por ende de lograr mayores tasas de crecimiento.

Siguiendo esta misma línea del debate, Hausmann et al (2007) vinculan el crecimiento de los países con el tipo de bienes que éstos exportan. De acuerdo con sus investigaciones, aquellas naciones que concentran parte importante de su oferta en los bienes que exportan los países desarrollados, tienen mejores y mayores tasas de crecimiento económico frente a las que se concentran en bienes primarios. Por lo anterior, concluyen que el tipo de bienes que conforman la canasta exportadora de una economía sí tiene incidencia en su crecimiento económico en el largo plazo.

Es así como la abundancia de recursos naturales no es condición suficiente para alcanzar altos niveles de crecimiento en las naciones. Por ello las recomendaciones de las investigaciones aplicadas alrededor del fenómeno de concentración exportadora en bienes primarios, advierten como la estrategia más urgente e importante el diversificar significativamente la canasta exportadora, a fin de dar paso al desarrollo y fortalecimiento de otros sectores estratégicos en el camino del desarrollo económico de las naciones, tales como la industria, las tecnologías blandas y duras, y de igual forma las tecnologías de la información y la comunicación (TIC), que permitan la creación de nuevas ventajas competitivas que impulsen los procesos de crecimiento y desarrollo económico de los países (Sachs \& Warner, 1997 Larraín et. al.,1999).

\section{Metodología}

El desarrollo de este trabajo investigativo tuvo como eje central el cálculo y posterior análisis del índice de concentración/diversificación exportadora por producto para el departamento de Santander durante el periodo 20002012, el cual se realizó en las siguientes etapas:

Etapa 1. Fundamentación teórica y estructuración de la metodología base para el cálculo del índice de concentración/diversificación exportadora por producto y por destino.

De acuerdo a la teoría económica del comercio internacional, para medir el grado de concentración/diversificación de una canasta exportadora, bien sea en términos de productos o destinos, es preciso calcular el Índice de Herfindahl Hirschmann o HH, denominado así en honor a sus desarrolladores, y utilizado en economía industrial para medir el grado de concentración de los mercados.

Dicho indicador, mide el grado de concentración exportadora al ponderar el peso de cada producto y país en el total de su comercio. Su cálculo se realiza de la siguiente forma:

$$
\text { (a) } I H H=\frac{\left[\sum_{j=1}^{n} \mathcal{P} i^{2}-\frac{1}{n}\right]}{1-\frac{1}{n}}
$$

Dónde: $P i=\frac{X i j}{X t i}$, indica la participación de mercado del país j en las exportaciones del país i en el total de sus exportaciones al mundo $(X T i)$. De esta forma, "se pondera el peso de cada producto y país en el total de su comercio de modo que si el valor exportado es reducido, tiene una influencia pequeña en el indicador final, y viceversa. Esto se controla al tomar el cuadrado delas participaciones de cada país" (Durán \& Álvarez, 2008).

La suma de los cuadrados de todas las participaciones se conoce como Índice de Herfindahl. "Al corregir por el número de observaciones, se adopta la metodología Herfindahl-Hirschmann, que permite comparar resultados 
entre diversos conjuntos de productos, países de destino de exportaciones o ambos al presentar los resultados en forma normalizada"(Durán \& Álvarez, 2008).

En lo referente al análisis, un índice HH superior a 18\% $(0,18)$ muestra un mercado concentrado. Entre el 10\% y $18 \%$, moderadamente concentrado. Mientras que uno que se encuentre entre el $0 \%$ y el10\%,refleja un mercado diversificado(Durán \& Álvarez, 2008).

Al ser la metodología más reconocida y de mayor uso para medir las variables de las que se ocupa este estudio, y teniendo como valor agregado, el hecho de que ha sido ampliamente aplicada por estudios empíricos de la Comisión Económica para América Latina y el Caribe (CEPAL), se adoptó la metodología HerfindahlHirschman, para medir el grado de concentración/diversificación exportadora del Departamento de Santander, tanto por productos como por destinos, para el periodo 2000-2012.

\section{Etapa 2. Recolección y Tratamiento de la} información

En concordancia con la metodología adoptada, se adquirió en el DANE la siguiente información, necesaria para el cálculo del índice $\mathrm{HH}$ para cada uno de los años de estudio:

1. Base de Datos de las exportaciones por producto del departamento de Santander para el periodo 2000-2012.

2. Base de Datos de las exportaciones por destino del departamento de Santander para el periodo 2000-2012.

Para el tratamiento de estos datos estadísticos, en primer lugar, se filtró la información por Código de Clasificación Internacional Industrial Uniforme (CIIU) revisión 2, para cada uno de los años. Posteriormente, para la medición del índice $\mathrm{HH}$ por producto, se procedió a organizar la base de datos de forma ascendente, totalizando las exportaciones por código CIIU; y, para el cálculo por destino, se construyó año a año, una tabla dinámica que totalizara las exportaciones del Departamento por país comprador. índice

Etapa 3 Aplicación metodológica y construcción del

Para el cálculo del Índice Herfindahl-Hirschmann por producto, la variable por la que se filtró en primera instancia la base de datos fue Código CIIU, revisión 2 adaptada para Colombia por la DIAN, categoría individual a cuatro dígitos, abarcando las 17 secciones de su estructura general y las actividades específicas de cada división. Lo anterior tuvo por objetivo medir, con la mayor precisión posible, el comportamiento de las exportaciones por producto del Departamento para cada año. Una vez filtrada la base de datos, se procedió a organizarla de forma ascendente y a totalizar las exportaciones de cada actividad registrada, respetando la clasificación CIIU.

Totalizadas las exportaciones por código CIIU, se aplicó la metodología HH para cada año, así:

$$
I H H=\frac{\left[\sum_{j=1}^{n} \mathcal{P} i^{2}-\frac{1}{n}\right]}{1-\frac{1}{n}}
$$

Donde: $P i=\frac{X i j}{X t i}$, representa la participación de mercado de cada actividad exportadora registrada de acuerdo a la división CIIU, en el total de las exportaciones del Departamento para cada año. Elevadas al cuadrado para mantener la proporción y normalizadas para efectos de análisis y comparación, teniendo en cuenta que $n$ representa el número total de actividades exportadoras por año. De esa forma se calculó el Índice Herfindahl-Hirschmann para cada uno de los años que abarcaron el periodo de estudio.

\section{Análisis de resultados}

A continuación se analizarán los resultados obtenidos de la aplicación de la metodología Herfindahl Hirschmann, para la medición del grado de concentración/diversificación exportadora por producto del departamento de Santander durante el periodo 20002012.

Figura 1. Índice Herfindahl Hirschmann por producto, Santander 2000-2012. Fuente: elaboración propia con base en datos DANE

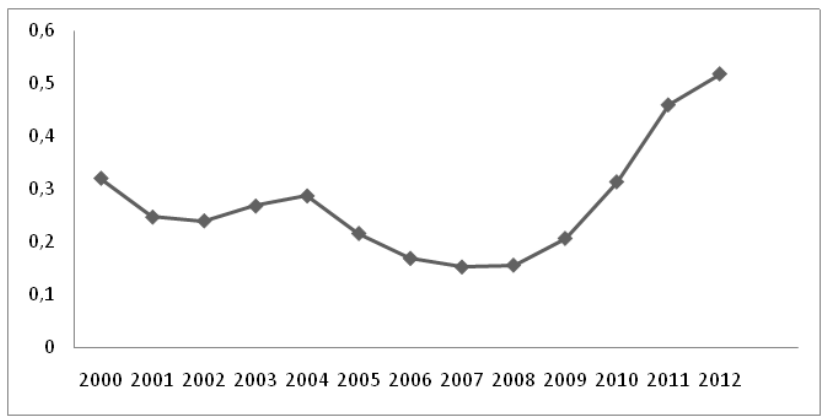

Fuente: Los autores

Al calcular el índice $\mathrm{HH}$ (o $\mathrm{IHH}$ ) por producto para Santander, se encuentra una tendencia creciente del grado de concentración que pasa de 0,32 en el año 2000 a 0,51 en 2012, tal como lo muestra la Figura 1. Lo anterior, deja ver claramente que el departamento ha concentrado considerablemente su canasta exportadora a lo largo de los 13 años que componen el periodo de estudio.

Que explica estos resultados? En primer lugar, dicha concentración, se dio de 2000-2005 en tres sectores: Agricultura, caza, silvicultura y pesca, Explotación de Minas y Canteras e Industria Manufacturera. Sin embargo, tal como se aprecia en la Figura 2, a partir del 2006, contrario a lo que se esperaría de una economía en crecimiento, el sector primario desaparece de la dinámica exportadora del departamento, sin que ningún otro surja 
como muestra del mayor desarrollo de la economía de la región. Así, desde 2006, se observa que la tendencia a la concentración de las exportaciones se profundiza aún más, al concentrase éstas en sólo dos sectores: Explotación de Minas y Canteras e Industria Manufacturera, comportamiento que se mantuvo así hasta2012.

Pese a lo anterior, durante el periodo 2000-2005, el sector de Agricultura, caza, silvicultura y pesca, que aún participaba en las exportaciones del departamento, creció en promedio a una tasa anual del 5,07\%, pasando de exportar USD\$17.101.571,00 en 2000, a USD\$ 23.020.062,04 en 2005, año después del cual sus ventas en el mercado internacional son prácticamente nulas.

Contrario a este comportamiento, el crecimiento promedio anual de las exportaciones de Minas y Canteras, fue del $61,71 \%$, durante todo el periodo de estudio, pasando de registrar ventas al extranjero por valor de USD\$ 34.800,00 en el 2000 a USD\$ 18.001.978, 16 en 2012.

La Industria Manufacturera, también registró una tasa de crecimiento promedio anual positiva del 16,92\%, pasando de exportar USD\$ 91.132.458,00 en el 2000, a USD\$ 695.724.469,02 al final del periodo de estudio.

Figura 2. Participación de los sectores en las exportaciones. Santander. 2000-2012.Fuente: elaboración propia con base en datos DANE

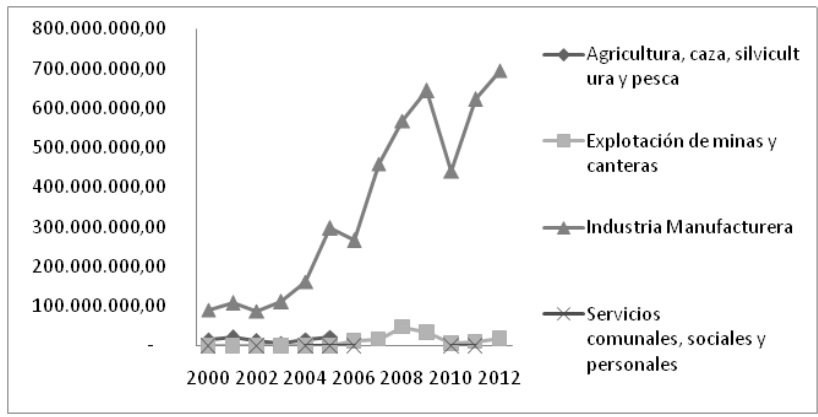

Fuente: Los autores

Según la Figura 2, los sectores con mayor participación en las exportaciones de Santander son: en primer lugar y muy por encima de los demás, industria manufacturera; en segundo lugar, explotación de minas y canteras, que ha mostrado una continuidad y un mayor crecimiento después del año 2005; en tercer lugar apareció hasta el año 2005 el sector primario representado por agricultura, caza, silvicultura y pesca, que como ya se dijo prácticamente desapareció del panorama exportador; y en último lugar, el sector de servicios comunales, sociales y personales que presenta dos periodos relevantes, uno del 2004 al 2006 donde significó un aporte al PIB regional, y un renacimiento durante el 2010 y 2011.

Los productos que componen la canasta exportadora más relevante, para el sector minas y canteras son básicamente los siguientes cinco:

1. Extracción de petróleo crudo y gas natural

2. Extracción de minerales de uranio y torio

3. Extracción de arenas y gravas silíceas

4. Extracción de minerales para la fabricación de abonos y productos químicos

5. Extracción de otros minerales no metálicos

Por su parte, para el sector de la Industria Manufacturera, se encuentran los siguientes 23 productos:

1. Carnes

2. Alimentos/bebidas

3. Café

4. Textiles

5. Calzado

6. Marroquinería

7. Tabaco

8. Artículos de Madera

9. Artículos de papel y cartón

10. Productos de la refinación del Petróleo

11. Derivados del Petróleo

12. Caucho

13. Vidrio

14. Hormigón, Cemento y Yeso

15. Hierro y Acero

16. Metal

17. Equipo Eléctrico, Médico y Quirúrgico

18. Maquinaria y Equipo

19. Relojes

20. Joyas

21. Vehículos y autopartes

22. Reciclaje

23. Productos Farmacéuticos y Medicamentos

A continuación, como lo muestra la tabla 1 , se puede observar la participación de los productos descritos del sector Minas y Canteras en el total de las exportaciones del departamento de Santander. 
Tabla 1. Productos que componen la canasta exportadora del Sector Minas y Canteras de Santander y su participación en el total de sus exportaciones

\begin{tabular}{|c|c|c|c|c|}
\hline Producto & $\begin{array}{l}\text { Valor } \\
\text { USD\$ } \\
\text { Año2000 }\end{array}$ & $\begin{array}{l}\text { \% Part. } \\
\text { Año } \\
2000\end{array}$ & $\begin{array}{l}\text { Valor } \\
\text { USD\$ } \\
\text { Año } 2012\end{array}$ & $\begin{array}{l}\text { \% Part. } \\
\text { Año } \\
2012\end{array}$ \\
\hline Extracción de minerales de uranio y de torio & & & $6.237 .480,08$ & 34,6 \\
\hline $\begin{array}{l}\text { Extracción de petróleo crudo y de gas natural, actividades } \\
\text { de servicios relacionadas con la extracción de petróleo y } \\
\text { gas, excepto las actividades de prospección }\end{array}$ & & & $5.795 .298,14$ & 32,2 \\
\hline Extracción de otros minerales no metálicos ncp & & & $5.721 .413,41$ & 31,8 \\
\hline Extracción y aglomeración de hulla (carbón de piedra) & & & $228.836,31$ & 1,3 \\
\hline $\begin{array}{l}\text { Extracción de minerales la fabricación de abono y } \\
\text { productos químicos }\end{array}$ & $34.800,00$ & 100 & $18.500,00$ & 0,1 \\
\hline Extracción de piedra, arena y arcillas comunes & & & 420 & 0,0 \\
\hline Extracción de arenas y gravas silíceas & & & 30,22 & 0,0 \\
\hline Total & $34.800,00$ & & 18.001.978,1 & \\
\hline
\end{tabular}

Fuente: Elaboración propia con base en DANE.

Se observa que para el año 2000, del sector de Minas y Canteras, el $100 \%$ de la participación se encontraba en la extracción de minerales, la fabricación de abono y productos químicos; esto apoyando la tendencia de crecimiento que se presentaba en este momento en el Sector primario del departamento, tendencia que cambia en 2012 donde la mayor participación del sector es de la extracción de minerales de uranio y de torio $(34,6 \%)$;extracción de petróleo crudo y de gas natural $(32,2 \%)$, actividades de servicios relacionadas con la extracción de petróleo y gas, excepto las actividades de prospección; y, extracción de otros minerales no metálicos $(31,8 \%)$, demostrando la fuerza que toma este sector liderando las exportaciones del departamento, donde ha resultado un elemento dinamizador el aporte de la Refinería de Petróleo en Barrancabermeja. Con respecto a la Industria manufacturera, los productos más exportados se muestran en la tabla 2.

Este sector muestra mayor dinamismo gracias al mismo fenómeno petrolero. Pues, tal como lo muestra la tabla 2, para el año 2000, los Derivados del petróleo aportaron la mayor fuente exportadora $(65,6 \%)$, equivalentes a USD \$59.751.947. Sin embargo, en 2012, su participación se redujo drásticamente al orden del $3,8 \%$, para una tasa de crecimiento negativa del $(6,2 \%)$ durante el periodo de estudio. Tal perdida en la participación no significó en ningún momento la pérdida de dinamismo del sector manufacturero de la región en este rubro, por el contrario, este descenso obedece al creciente auge exportador que durante el periodo de estudio tuvieron los a Productos de la refinación del Petróleo, que en 2012 representaron el 73,8\% de las ventas manufactureras al extranjero del departamento, lo que significó una entrada de USD\$ 513.386.997 en ese año para la Región, pasando de exportar en el año 2000 un valor USD $\$ 1.278 .418,00$ que tan solo obedecía al $1,4 \%$ de las exportaciones del sector, consolidando así una tasa de crecimiento de $58,6 \%$ para todo el periodo de estudio, que se explica, en parte, por el fortalecimiento de la Refinería de Barrancabermeja y la Inversión Extranjera Directa que ha llegado al departamento para este fin.

'Ídem.

Cursivas de los autores para resaltar el nombre de la partida arancelaria. 
Tabla 2. Productos que componen la canasta exportadora de la industria manufacturera de Santander y su participación en las exportaciones del sector

\begin{tabular}{|c|c|c|c|c|c|}
\hline Producto & $\begin{array}{l}\text { Valor USD\$ } \\
\text { Año } 2000\end{array}$ & $\begin{array}{l}\% \text { Part. } \\
\text { Año } 2000\end{array}$ & $\begin{array}{l}\text { Valor USD\$ Año } \\
2012\end{array}$ & $\begin{array}{l}\% \text { Part. } \\
\text { Año } 2012\end{array}$ & $\begin{array}{llll}T & \text { a } & \mathbf{s} & \mathbf{a} \\
\text { Crecimiento }\end{array}$ \\
\hline Carnes & $3.852,00$ & 0,004 & $13.021 .105,50$ & 1,872 & 86,835 \\
\hline $\begin{array}{l}\text { Ptos. Farmacéuticos y } \\
\text { Medicamentos }\end{array}$ & 140,00 & 0,000 & $145.056,65$ & 0,021 & 70,590 \\
\hline Hierro y Acero & $21.017,00$ & 0,023 & $9.861 .427,20$ & 1,417 & 60,506 \\
\hline $\begin{array}{l}\text { Pdctos. Refinación de } \\
\text { Petróleo }\end{array}$ & $1.278 .418,00$ & 1,403 & $513.386 .997,02$ & 73,792 & 58,595 \\
\hline $\begin{array}{l}\text { Equipo Eléctrico, } \\
\text { Médico y Quirúrgico }\end{array}$ & $26.894,00$ & 0,030 & $1.316 .076,69$ & 0,189 & 34,887 \\
\hline Metal & $117.640,00$ & 0,129 & $3.502 .531,83$ & 0,503 & 29,829 \\
\hline Caucho & $11.534,00$ & 0,013 & $259.612,02$ & 0,037 & 27,065 \\
\hline Joyas & $54.209,00$ & 0,059 & $419.378,60$ & 0,060 & 17,044 \\
\hline Vidrio & $38.225,00$ & 0,042 & $267.360,07$ & 0,038 & 16,140 \\
\hline Art. Madera & $229.388,00$ & 0,252 & $1.594 .082,13$ & 0,229 & 16,082 \\
\hline Vehículos y Autopartes & 3.494 .905 & 3,835 & $16.863 .082,13$ & 2,424 & 12,870 \\
\hline Tabaco & $819.692,00$ & 0,899 & $3.427 .949,88$ & 0,493 & 11,635 \\
\hline Alimentos/Bebidas & 1.247 .047 & 1,368 & $3.860 .179,72$ & 0,555 & 9,081 \\
\hline Maquinaria y Equipo & 3.311 .385 & 3,634 & $10.238 .179,57$ & 1,472 & 9,071 \\
\hline Calzado & 3.700 .683 & 4,061 & $6.295 .821,95$ & 0,905 & 4,172 \\
\hline Marroquinería & $453.736,00$ & 0,498 & $525.277,40$ & 0,076 & 1,133 \\
\hline Textiles & 14.091 .915 & 15,463 & $14.723 .465,05$ & 2,116 & 0,338 \\
\hline Art. Papel y Cartón & $254.628,00$ & 0,279 & $224.559,72$ & 0,032 & $-0,962$ \\
\hline Derivados del Petróleo & $59.751 .947,00$ & 65,566 & $26.160 .696,67$ & 3,760 & $-6,156$ \\
\hline Otros & 2.223 .915 & 2,440 & $689.627,56$ & 0,099 & $-8,613$ \\
\hline $\begin{array}{l}\text { Hormigón, Cemento y } \\
\text { Yeso }\end{array}$ & $1.288,00$ & 0,001 & 0,00 & 0,000 & $-68,834$ \\
\hline Café & & 0,000 & $68.942 .001,66$ & 9,909 & \\
\hline Relojes & & 0,000 & & 0,000 & \\
\hline Reciclaje & & 0,000 & & 0,000 & \\
\hline Total & 91.132.458 & 100,000 & $695.724 .469,02$ & 100,000 & \\
\hline
\end{tabular}

Fuente: Elaboración propia con base en DANE.

Es de resaltarla tasa de crecimiento, para el periodo de estudio, de las exportaciones de las Carnes en el departamento, que fue del $86,9 \%$; los Productos Farmacéuticos y Medicamentos (70,6\%); Hierro y Acero (60,5\%); Equipo Eléctrico, Médico y Quirúrgico (34,9\%); Metal (29,8\%); y, Caucho (27,1\%), que no corresponden al rubro de exportaciones tradicionales de Santander pero que revelan un aprovechamiento de las externalidades positivas alrededor del fenómeno petrolero y el desarrollo urbanístico de los principales municipios del departamento, para atender nuevos nichos de mercado.
Dentro de los productos que se cuentan como tradicionales en la vocación exportadora del departamento, se encuentran los siguientes datos relevantes: el sector de Joyería presenta una tasa de crecimiento del $17 \%$ a lo largo del periodo de estudio, ubicándose en el octavo lugar de importancia dentro de los productos que componen la canasta exportadora del sector manufacturero de Santander, pasando de un valor exportado de USD \$54.209,00 en el 2000 a USD $\$ 419.378,60$ en 2012, tal como lo muestra la Figura 3 
Figura 3. Variación Productos de Joyería en el total de las exportaciones de la Industria Manufacturera, 2000-2012. USD. Fuente: elaboración propia con base en DANE.

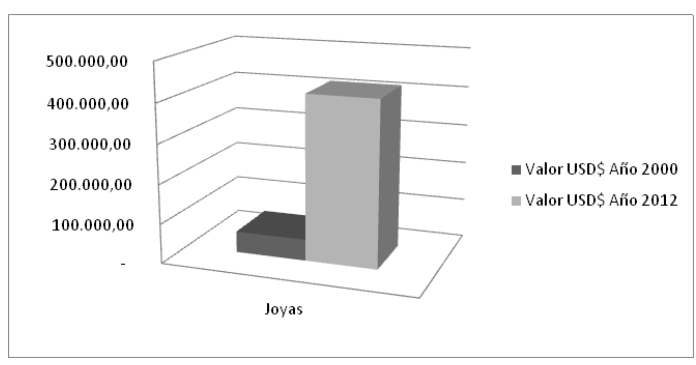

Fuente: Los autores

Para el caso del Café, su incremento ha sido sustancial pasando de no participar en el año 2000 en las exportaciones del departamento a representar el 9,9\% de las exportaciones de la Industria Manufacturera en el año 2012, que en términos monetarios se traduce en unos ingresos para el departamento por USD\$68.942.001 (Ver Figura 4)..

Figura 4. Variación de la Producción de Café en el total de las exportaciones de la Industria Manufacturera, 20002012. USD. Fuente: elaboración propia con base en DANE.

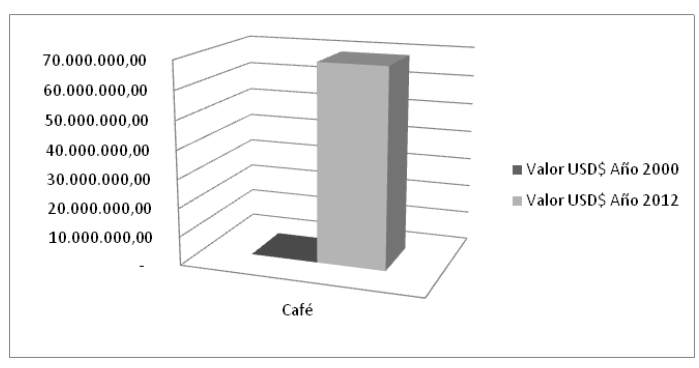

Figura: Los autores

La Producción de textiles, es una de las actividades que sorprende por su participación decreciente en el total de exportaciones del sector manufacturero del departamento, pasando de $15,5 \%$ en 2000 a tan solo un $2,1 \%$ en 2012 . Sin embargo, como lo muestra la Figura 7, ha crecido en términos monetarios, pasando de exportar un valor de USD\$14.091.915 en 2000 a USD\$14.723.465.

Figura 5.Variación Producción de Textiles en el total de las exportaciones de la Industria Manufacturera, 2000-2012. USD. Fuente: elaboración propia con base en DANE.

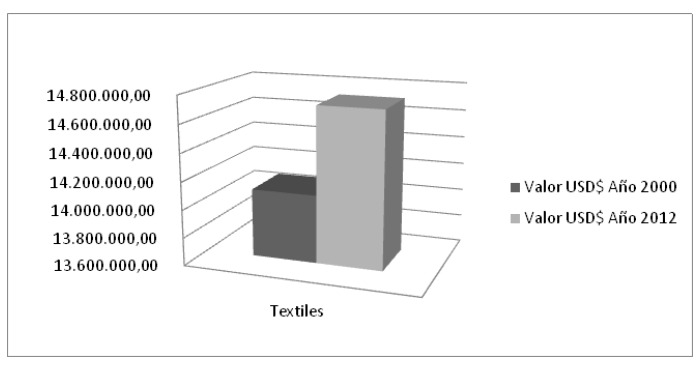

Figura: Los autores
Otra de las actividades tradicionales del departamento, la de la Producción de Calzado, como lo muestra la Figura 6, presenta una baja significativa de participación en el sector, pasando de $4,1 \%$ en 2000 a $0,9 \%$ en 2012, aunque en términos monetarios se presenta un incremento en este mismo periodo pasando de USD\$3.700.683 a USD\$6.295.821 en términos de valor exportado.

Figura 6. Variación de la Producción de Calzado en el total de las exportaciones de la Industria Manufacturera, 20002012. USD. Fuente: elaboración propia con base en DANE.

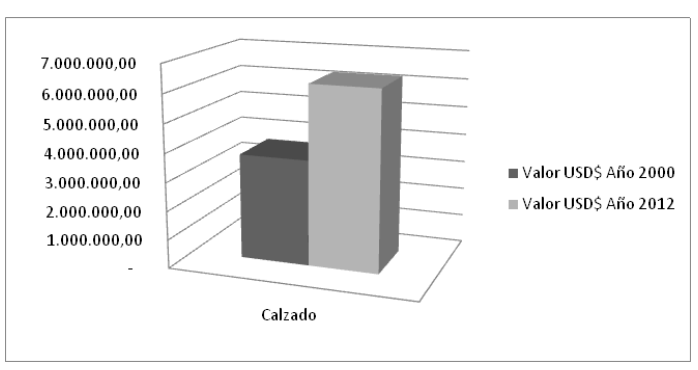

\section{Fuente: Los autores}

Otro sector que participa en las exportaciones del departamento, aunque de forma leve e intermitente, es el de Servicios sociales, comunales y personales, cuyo producto exportable son las actividades teatrales y musicales y otras actividades artísticas. Sin embargo, su participación se dio entre los años 2004-2006 y 2010-2011, por lo que no representa una partida representativa para el análisis.

Los demás sectores de la economía: Suministro de electricidad, gas y vapor; Obras hidráulicas y suministro de agua; Construcción; Comercio al por mayor y al por menor, y Restaurantes y hoteles; Transportes, almacenamiento y comunicaciones; y, Establecimientos financieros, seguros, bienes inmuebles y servicios prestados a las empresas, no registran participación alguna en las exportaciones del departamento durante los 13 años que componen el periodo de estudio.

\section{Conclusiones}

El IHH por producto para Santander, muestra claramente que el departamento ha concentrado considerablemente su canasta exportadora a lo largo de los 13 años que componen el periodo de estudio, particularmente desde 2006, año en el que este fenómeno se profundiza aún más, al concentrarse la oferta exportable del departamento en sólo dos sectores: Explotación de Minas y Canteras e Industria Manufacturera, comportamiento que se mantuvo así hasta2012.

Los productos que componen la canasta exportadora para el sector minas y canteras, provienen de actividades meramente extractivas de recursos naturales con poco o ningún grado de transformación $\mathrm{y} / \mathrm{o}$ aporte de valor agregado entre las que se cuentan: Extracción de petróleo crudo y gas natural; de minerales de uranio y torio; de arenas y gravas silíceas; de minerales para la fabricación de abonos 
y productos químicos; y de otros minerales no metálicos. Todas ellas altamente correlacionadas con el boom minero energético que se presentó en la región desde 2006 hasta el último año de estudio.

En la Industria Manufacturera, segundo sector exportador de Santander, los siguientes productos componen su oferta exportable: Carnes, Alimentos/bebidas, Café, Textiles, Calzado, Marroquinería, Tabaco, Artículos de Madera, Artículos de papel y cartón, Productos de la refinación del Petróleo, Derivados del Petróleo, Caucho, Vidrio, Hormigón, Cemento y Yeso, Hierro y Acero, Metal, Equipo Eléctrico, Médico y Quirúrgico, Maquinaria y Equipo, Relojes, Joyas, Vehículos y autopartes, Reciclaje, Productos Farmacéuticos y Medicamentos. La mayoría de estos productos, corresponden a las exportaciones ya tradicionales del departamento y cuentan con muy bajo nivel de transformación y aporte de valor agregado, razón por la cual preocupa una concentración de las exportaciones en esta canasta de bienes que no promueven el desarrollo de procesos de innovación tecnológica que irradien el desarrollo de otros sectores de la economía santandereana.

El fenómeno de concentración exportadora por producto en Santander, refleja un estado de involución sistemática de la Política Comercial del departamento, que ha permitido que la vocación exportadora de la región se reduzca a actividades de por sí ya tradicionales en el departamento, dejando de lado, la innovación proveniente de la incursión en otros sectores económicos de mayor transformación, valor agregado y complejidad tecnológica, tal como lo advierten los trabajos de Sachs \& Warner (1997; Larraín et al, (1999)Krugman, (1987) y Grossman \& Helpman (1991). Por tanto se sugiere el diseño y la ejecución de una política pública regional que busque cambiar esta realidad y el aprovechamiento de las políticas nacionales diseñadas para tal fin.

\section{Referencias}

James, R., Acemoglu, D \& Johnson, S.(2003). “An African Success Story: Botswana" In: Search of Prosperity: Analytic Narratives on Economic Growth. edited by Dani Rodrik, Princeton: Princeton University Press.

Antón, A., \& Villegas, A. (2010). El papel de la tasa de interés real en el ciclo económico de México. México D.F.:

Basal, J., Licandro, G. \& Rodríguez, H. (2011). Una nueva metodología de medición de la rentabilidad de la industria exportadora uruguaya. Banco Central del Uruguay.

Bonaglia, F. \& Fukasaku, K. (2003). Export diversification in low income countries. Documento de trabajo 209, OECD Development Centre.

Bonilla Uribe, M. J., \& Martínes Gallego, M. A. (2009). Análisis de la metodología para evaluar la competitividad:
Caso Foro económico Mundial y realidad empresarial colombiana. Bogotá: Universidad del Rosario.

CEPAL. (2009). Comercio internacional y desempeño económico. Indicadores seleccionados para economías pequeñas. México D.F.: Autor.

Delgado, L. (2009). Las políticas públicas. El ciclo de las políticas públicas. Clases de políticas públicas. Eficacia, legalidad y control. Indicadores de gestión. En L. Delgado, Documentación sobre gerencia pública, del Subgrupo A2, Cuerpo Técnico, especialidad de Gestión Administrativa, de la Administración de la Junta de Comunidades de Castilla-La Mancha (págs. 4-9). La Mancha: Junta de Comunidades de Castilla-La Mancha. Consejería de Administraciones Públicas. Escuela de Administración Regional.

De Ferranti, D., Lederman, D., Maloney, W., Perry, G. (2001). From Natural Resources to the Knowledge Economy: Trade and Job Quality. Advance Conference Edition. Banco Mundial.

Di Paula, C., Paladino, L., Plottier, C., Silveira, L. (2008). Políticas activas para la promoción de exportaciones. Exportación Inteligente. Un puente entre el sector exportador y la Academia. Jornada Académica 2008, pp. $39-128$.

Durán Lima, J. E., \& Alvarez, M. (2008). Indicadores de comercio exterior y política comercial: mediciones de posición y dinamismo comercial. Santiago de Chile: Naciones Unidas.

Durán Lima, J. E., \& Alvarez, M. (2011). Manual del comercio exterior y política comercial. Santiago de Chile: Naciones Unidas.

Esquivel, G., \& Larraín, F. (2001). ¿Cómo atraer Inversión Extranjera Directa? Mexico: Universidad de Harvard.

Fondo Monetario Internacional. (2002). Programación financiera: Métodos y aplicación al caso Colombia. Autor.

Garay S., Luis Jorge.(2000) Colombia: estructura industrial e internacionalización 1967-1996. Bogotá: Banrepublica

Gobernación de Santander. (2012) Plan Departamental de Desarrollo 2012-2015: "Santander en Serio, El Gobierno de la Gente". Bucaramanga. 2012.

Grossman, G. \& Helpman, E. (1991). "Quality Ladders in the Theory of Growth", the Review of Economic Studies, Vol. 58, No. 1.(Jan., 1991), pp. 43-61.

Hausmann, R. y Rodrik, D. (2003). "Economic 
development as self-discovery". Journal of Development Economics, 72, 2, pp. 603-633.

Hausmann, R., Hwang, J., Rodrik, D., (2007). "What you export matters". Journal of Economic Growth, 12, 1, pp. $1-25$.

Hernandez Sampieri, R., Fernández Collado, C., \& Baptista Lucio, P. (1997). Metodología de la Investigación. México: Mc Graw Hill.

Herzer, D. y Nowak-Lehmann, F. (2006). "What does export diversification do for growth? An econometric Analysis". Applied Economics, 38, 15, pp. 1825-1838.

Imbs, J. y Waczriag, R. (2003). "Stages of Diversification”. American Economic Review, 93, 1, pp. 63-86.

Instituto Municipal de Empleo de Bucaramanga. (2013). Industria del calzado y su visualización internacional.Bucaramanga.

Krugman, Paul,(1987). "The narrow moving band, the Dutch disease, and the competitive consequences of Mrs. Thatcher: Notes on trade in the presence of dynamic scale economies," Journal of Development Economics, Elsevier, vol. 27(1-2), pages 41-55, October.

Larraín, F., J. Sachs, y A. Warner (1999): A structural analysis of Chile's long term growth: history, prospects and policy implications, mimeo, Min. de Hacienda, Chile.

Lederman D. y W. Maloney (2001): Open questions about the link between natural resources and economic growth: Sachs and Warner revisited, mimeo, World Bank, Washington D.C.

Lederman, D. y Maloney, W. (2002). Open questions about the Link between Natural Resources and Economic Growth: Sachs and Warner Revisited. Documento de trabajo 141, Banco Central de Chile.

Lederman, D., Olarreaga, M. \& Perry, G. (eds.) (2007). "Latin America's Response to China and India: Overview of Research Findings and Policy Implications", Lederman, D., Olarreaga, M. \& Perry, G. (eds.) (2009).

Lederman, D., Olarreaga, M. \& Perry, G. (eds.) (2009). "China's and India's Challenge to Latin America. Opportunity or Threat?" The World Bank, Washington D.C.

Marulanda, J.(2010). Panorama de la inversión extranjera en Colombia. Documentos no. 81 de la escuela. Medellín: Escuela Nacional Sindical.

Márquez Escobar, P. (2001). Economía de la inversión extranjera en Colombia.Editorial McGraw Hill. Bogotá.
Mehlum, H., Moene, K. \& Torvik, R., (2006). "Cursed by Resources or Institutions?" The World Economy, Wiley Blackwell, vol. 29(8), pages 1117-1131, 08.

Meller, P. (2002): Dilemas y Debates en Torno al Cobre. Santiago: Dolmen Ediciones.

Meller, P., \& Moser, R. (2012). Análisis de las ex portaciones e n latinoamérica diversificación/concenctración. Santiago: CAF-CIEPLAN.

Ministerio de Comercio, Industria y Turismo (2013) Comercio Exterior. Acuerdos Comerciales. 2013.

Ministerio de Comercio, Industria y Turismo. (2010). Importacionescolombianas: hacia la modernidad o el estancamiento.

Ministerio de Comercio, Industria y turismo. ( 2010). Dinámica exportadora de Colombia (2002-2009).

Muller, P. (1998). "La producción de las políticas públicas". INNOVAR, revista de ciencias administrativas y sociales, 66-67.

Municipio de Bucaramanga.(2012) Plan de Desarrollo 2012-2015: "Bucaramanga, Capital Sostenible". Bucaramanga.

Prebisch, R. (1950). The Economic Development of Latin America and its Principal Problems. Reimpreso en Economic Bulletin for Latin América, 7, 1, 1962.

Presidencia de la República. (2008). Diagnóstico Socioeconómico del Departamento de Santander. Bogotá: Autor.

Quiroz, S. (2008). Tendencia de la inversión extranjera directa en México 2005-2008.México D.F.: Revista Economía Actual.

Robinson, J., Torvik, R. \& Verdier, T. (2006)."Political foundations of the resource curse," Journal of Development Economics, Elsevier, vol. 79(2), pages 447-468.

Sachs, J. y Warner, A. (1997). Natural Resource Abundance and Economic Growth- Center for International Development. Harvard Institute for International Development, Harvard University.

Singer, H. (1950). US Foreign Investment in Underdeveloped Areas: The Distribution of Gains between Investing and Borrowing Countries. American Economic Review, 40, 2, pp. 473-485.

Torvik, R., (2001): "Learning by doing and the Dutch disease". European Economic Review 45, 285-306. 
Vásquez, S., Plottier, C.,y Zuasnabar, A. (2011). Diversificación y Dinamismo Exportador, ¿Todas son buenas noticias? Jornadas Académicas. Universidad Católica del Uruguay.

Velásquez, R. (2009). Hacia una nueva definición del concepto de "política pública". Desafíos, 156.
World Bank (2002). "From Natural Resources to the Knowledge Economy", World Bank Latin American and Caribbean Studies, Washington D.C. 